\section{Cécile Butor \\ Anne Couëdel- \\ Courteille \\ Alain Venet \\ Jean-Gérard Guillet}

\title{
Immunité locale et vaccination
}

L'organisme est protégé contre l'entrée des micro-organismes au niveau des muqueuses par toute une série de mécanismes dont les premiers sont non spécifiques, passifs (étanchéité de l'épithélium, polymères glucidiques, glycoprotéines) ou actifs (défensines, réactions inflammatoires). Le système immunitaire muqueux spécifique se met en place lorsque les premiers mécanismes de défense ont échoué. Dans l'intestin, la réponse immune muqueuse débute dans l'épithélium et les follicules lymphoïdes. Les lymphocytes B activés poursuivent leur maturation dans les ganglions drainant la muqueuse intestinale, passent dans la circulation générale et retournent, entre autres, à leur muqueuse d'origine, qui apparaît comme un site de domiciliation privilégié. Ils s'y différencient en plasmocytes qui sécrètent les anticorps spécifiques sous forme d'IgA solubles. Une réponse immunitaire à médiation cellulaire se développe aussi par l'intermédiaire des lymphocytes $T$ cytotoxiques intra-épithéliaux. De nombreuses stratégies vaccinales, visant à protéger les muqueuses, ont pour but de stimuler elles aussi la sécrétion locale d'IgA spécifiques.

C. Butor: chercheur post-doctoral ANRS A. Couēdel-Courteille: étudiante doctorale. A. Venet: chargé de recherche à l'Inserm. J.G. Guillet : directeur de recherche à l'Inserm. Inserm U. 152, Institut Cochin de génétique moléculaire, 22 , rue Méchain, 75014 Paris, France.

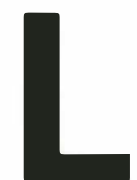

e système immunitaire muqueux (figures 1 et 2) est particulièrement complexe. Il permet la mise en place d'une immunité locale utilisant aussi bien la réponse immunitaire adaptative que non spécifique pour constituer une "barrière" efficace contre l'entrée des micro-organismes pathogènes dans l'organisme. Le système lymphoïde de l'intestin contient à lui seul plus de cellules immunitaires que tout le reste de l'organisme [1-3]. La réponse immunitaire locale est induite au niveau des follicules lymphoïdes et de leur épithélium associé. Les lymphoblastes poursuivent leur maturation dans les ganglions drainant les muqueuses et repeuplent ensuite l'épithélium et le chorion des muqueuses, où ils se différencient en effecteurs. L'immunité systémique n'intervient que lorsqu'une série de mécanismes de protection de l'organisme contre l'envahissement par les micro-organismes s'est révélée inefficace.

\section{Barrière épithéliale}

Les épithéliums de recouvrement séparent le milieu intérieur de l'organisme du milieu extérieur. Ces épithéliums forment une barrière, mais ils organisent également les interactions avec le milieu extérieur. Les différences dans l'état physique du milieu avec lequel ils interagissent 


\section{RÉFÉRENCES}

1. Cerf-Bensussan N, Guy-Grand D. Organisation du système lymphoïde associé à l'intestin. Rev Fr Allergol 1993; 33: 197-203.

2. McGhee JR, Mestecky J, Dertzbaugh MT, Eldridge $\mathrm{JH}$, Hirasawa $\mathrm{M}$, Kiyono $\mathrm{H}$. The mucosal immune system: from fundamental concepts to vaccine development. Vacine 1992; 10 : 75-88.

3. Staats HF, Jackson RJ, Marinaro M, Takahashi I, Kiyono H, McGhee JR. Mucosal immunity to infection with implications for vaccine development. Curr Op Immunol $1994 ; 6: 572-83$.

4. Varki A. Biological roles of oligosaccharides: all of the theories are correct. Glyco biology $1993 ; 3: 97-130$.

5. Pattus F. Les armes peptidiques de la défense antimicrobienne: un champ d'investigation en pleine expansion. médecine/sciences $1992 ; 8: 420-2$.

6. Nicolas P, Mor A, Delfour A. Les peptides de la défense antimicrobienne des vertébrés. médecine/sciences $1992 ; 8$ : 423-31.

7. Keystone Symposium. Mucosal immunity new strategies for protection against vira and bacterial pathogens. / Cell Biochem 1995 (suppl 19A) : 231-67.

Voir particulièrement les abstracts de Svanborg et al. (J1-006), Pringault et al. (J1-003) Pascopella (présentation orale), Zychlinsky et al. (J1-148), Mestecky et al. ([1-010), Czerkinsky (J1-late abstract), Levine (J1-019), Palese et García-Sastre (J1-021).

8. Neutra MR, Kraehenbühl JP. The role of transepithelial transport by $M$ cells in microbial invasion and host defense. I Cell S $r i$ 1993; (suppl. 17) : 209-15.

9. MacDermott RP, Lichtenstein GR, Izutani $\mathrm{R}$, Muraki T. Anomalies du système immunitaire de la muqueuse au cours des maladies inflammatoires de l'intestin. médecine/ sciences $1993 ; 9: 853-9$.

10. Farstad IN, Halstensen TS, Fausa $O$ Brandtzaeg P. Heterogeneity of M-cell-associated B and $T$ cells in human Peyer's (sec, humide, liquide), dans leur spécialisation fonctionnelle et dans les forces mécaniques qu'ils subissent font qu'ils adoptent l'une des deux grandes structures épithéliales: épithéliums simples, épithéliums stratifiés. L'ensemble des couches cellulaires d'un épithélium stratifié est conceptuellement et fonctionnellement équivalent à la monocouche cellulaire d'un épithélium simple. La face muqueuse, ou apicale, de l'épithélium a une constitution lipidique et protéique différente de la face séreuse, ou basolatérale, exposée au milieu intérieur.

Tout micro-organisme doit traverser l'un ou l'autre de ces épithéliums pour infecter son hôte. Trois stratégies générales le permettent: profiter d'une lésion dans l'épithélium, infecter les cellules épithéliales, se faire transporter à travers l'épithélium sans infecter les cellules épithéliales (figure 3). De nombreuses variantes de ces stratégies de base sont exploitées aussi bien par les bactéries que par les virus ou les parasites. Afin d'éviter cette colonisation par des agents pathogènes, de multiples systèmes de défense complémentaire des épithéliums sont à la disposition de l'organisme. Les différences de structure, d'état physique du milieu " extérieur", et de types d'antigènes auxquels sont exposés les épithéliums, font que les systèmes de défense sont modulés en fonction du site. Les polymères glucidiques et les glycoprotéines constituent une ligne de défense essentielle des muqueuses, souvent méconnue [4]. Le mucus englue les micro-organismes, forme une barrière physique qui les maintient à distance et facilite leur glissement à la surface de la muqueuse. Le glycocalyx a un rôle similaire à la surface des cellules épithéliales. Enfin, des modifications des sucres présents sur des glycoconjugués de surface permettent de masquer les récepteurs de certains pathogènes, et/ou de les transformer en récepteurs pour des pathogènes moins virulents. L'O-acétylation des acides sialiques les convertit de récepteurs pour influenza A et influenza B en récepteurs pour influenza $\mathrm{C}$, beaucoup moins virulents. La sensibilité aux infections urinaires par différentes souches d'Escherichia coli est déterminée par les glycolipides exprimés dans les voies urinaires.
Les défensines, peptides à activité bactéricide ou viricide, sont sécrétées par des cellules épithéliales spécialisées. Elles ont fait récemment l'objet d'articles de synthèse dans médecine/ sciences, auquels nous renvoyons le lecteur $[5,6]$.

La réaction inflammatoire conduit à la transmigration de leucocytes (en particulier des polynucléaires neutrophiles) dans la lumière des muqueuses. Dans ce contexte, il est remarquable de noter l'expression par les cellules intestinales des molécules du complexe majeur d'histocompatibilité de classe II (CMH II) sur le domaine apical ( $m / s n^{\circ} 5$, vol. $\left.7, p .512\right)$. Théoriquement, les cellules épithéliales pourraient donc présenter des antigènes à des lymphocytes $\mathrm{T}$ auxiliaires présents dans la lumière intestinale. Le rôle d'une telle présentation reste mystérieux. La transmigration peut être déclenchée par la production de cytokines (par exemple, dans les infections urinaires IL8 [7] par les cellules épithéliales). Cette production, en réponse à d'autres cytokines ou à l'exposition à des bactéries pathogènes, est mise en évidence dans des systèmes de plus en plus nombreux, in vitro ou in vivo. Elle reste faible comparée à la production de cytokines par les cellules immunitaires de la muqueuse, mais pourrait jouer un rôle primordial dans les mécanismes immunitaires à l'origine de la réponse immunitaire non spécifique. Par ailleurs, certaines cytokines (interféron par exemple) sont capables d'induire un état de résistance à l'infection des cellules épithéliales. Ces facteurs non spécifiques agissent dans les minutes ou les heures qui suivent l'exposition à un agent pathogène. Ils permettent habituellement à l'organisme de contenir une infection pendant le temps nécessaire à la mise en place d'une réponse immunitaire spécifique, ce qui peut prendre quelques jours.

\section{Induction de la réponse spécifique: les sites inducteurs}

Le transport des antigènes intacts de la membrane apicale exposée à la lumière du tractus gastro-intestinal jusqu'à la surface basolatérale, là où l'antigène va pouvoir interagir avec les cellules du système réticulo-histio- 


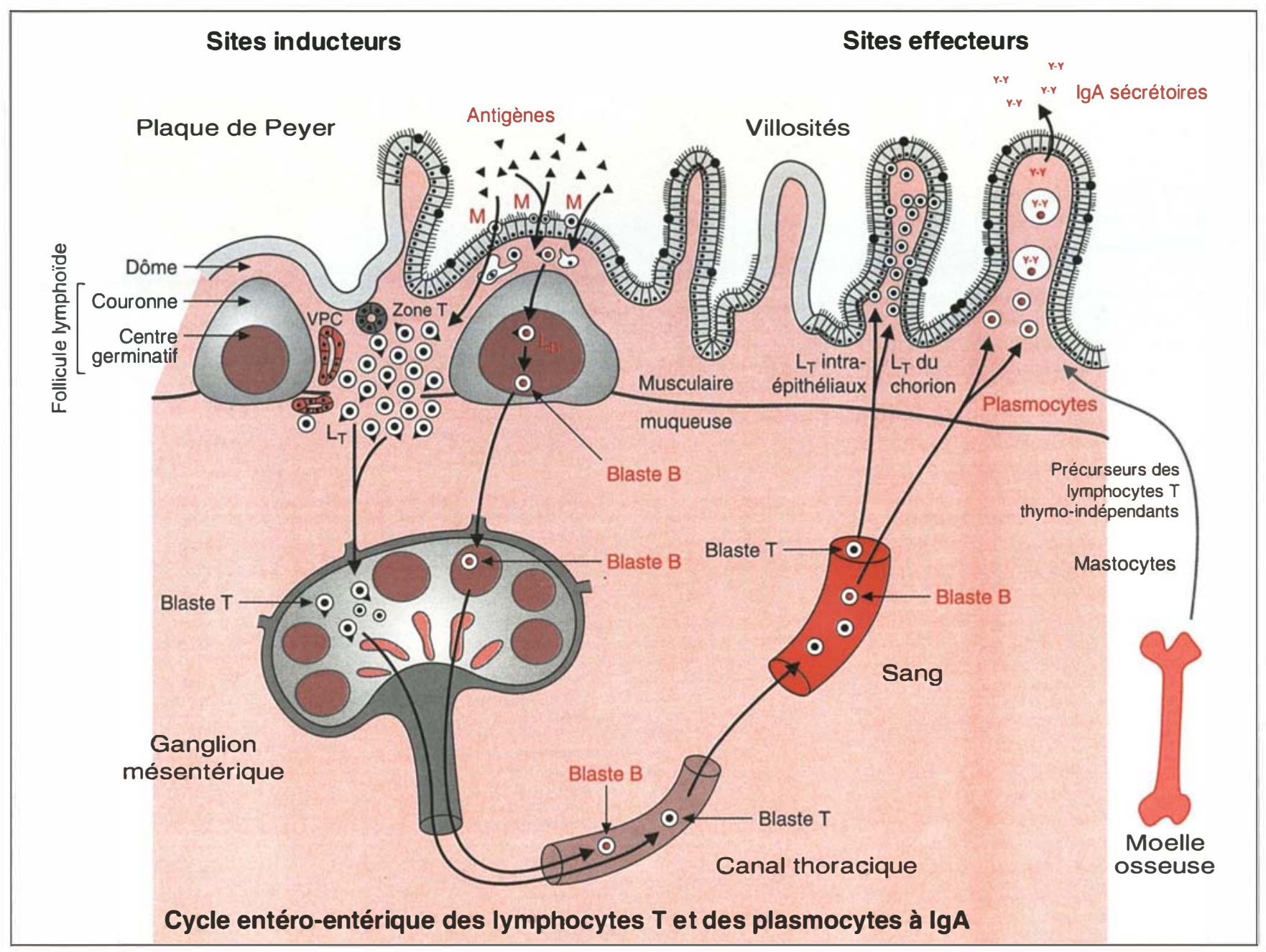

Figure 1. Système immunitaire muqueux associé à l'intestin. Ce système est constitué à la fois par le cycle entéroentérique des lymphocytes T et des plasmocytes à IgA (flèches noires), et par des effecteurs cellulaires (lymphocytes $T$ intraépithéliaux thymo-indépendants, mastocytes) dont les précurseurs proviennent directement de la moelle osseuse (flèche grise). Les sites inducteurs du cycle entéro-entérique regroupent les follicules lymphoïdes et leur épithélium associé (regroupés en plaques de Peyer dans l'intestin grêle) ainsi que les ganglions mésentériques. Le transport des antigènes s'effectue par les cellules $M$. Les lymphocytes de la poche intraépithéliale représentent une extension du follicule lymphoïde sous-jacent. Les précurseurs B activés commencent leur maturation dans les centres germinatifs des follicules lymphoïdes, puis migrent dans les centrés germinatifs des ganglions mésentériques. Les précurseurs $T$ activés commencent leur maturation dans les ganglions mésentériques. Blastes $B$ et blastes $T$ rejoignent la circulation générale par l'intermédiaire du canal thoracique. Ils se rendent ensuite dans les sites effecteurs, constitués par les villosités de l'intestin. Les blastes B se différencient en plasmocytes à lgA dans le chorion. Les blastes T migrent, soit dans le chorion, soit dans l'épithélium (essentiellement des lymphocytes T CD8 ${ }^{+}$), et achèvent leur différenciation en lymphocytes $T$ sous l'influence de facteurs locaux. $M:$ cellules $M ; L_{B}:$ lymphocytes $B ; L_{T}$ : lymphocytes $T$; VPC : veinule post-capillaire. (Modifié d'après [21].)

cytaire pour pouvoir induire une réponse immunitaire, est réalisé par des cellules épithéliales spécialisées, les cellules $\mathrm{M}[8,9]$. Ces cellules n'expriment pas les molécules du CMH Il, et ne semblent donc pas avoir une fonction de présentation de l'antigène. L'origine de la cellule $M$ n'est pas encore résolue. On peut suivre les précurseurs des cellules $\mathrm{M}$ à partir des $\mathrm{m} / \mathrm{s} n^{\circ} 5$, vol.11, mai 95 cryptes intestinales, ce qui est compatible avec un programme de différenciation spécifique. Récemment, cependant, il a été possible d'induire la différenciation in situ de cellules épithéliales classiques en cellules $\mathrm{M}$ in vivo ou in vitro en les cocultivant avec des lymphocytes provenant de plaques de Peyer sur leur face basolatérale [7]. Les cellules $\mathrm{M}$ de différents sites ne sont pas identiques et se spécialisent en fonction du site. On sait également maintenant qu'il y a une spécificité d'espèce des cellules $M$. Les salmonelles, par exemple, ne sont pathogènes que pour l'espèce dans laquelle les cellules $\mathrm{M}$ les transportent [7]. Il devient donc difficile de transposer des résultats obtenus sur les cellules M d'une espèce à l'autre. 
Figure 2. Sites inducteurs et sites effecteurs du système immunitaire digestif du macaque. A. Épithélium associé à un follicule rectal. Cet épithélium est constitué de cellules épithéliales et de cellules $M$. De nombreux Iymphocytes sont visibles dans les poches intraépithéliales des cellules $M$. Les cellules situées sous cet épithélium sont majoritairement des cellules immunitaires llymphocytes $T$ et $B$ et macrophages), qui constituent la zone appelée le dôme. $B$. Vue générale d'une muqueuse rectale. On distingue nettement un follicule lymphoïde (F) dont la densité cellulaire contraste avec celle de la lamina propria (LP). L'épithélium de recouvrement $(E)$ forme de larges villosités, qui disparaissent au-dessus du follicule où l'épithélium est différent. Le follicule représenté ici est particulièrement important et pénètre profondément dans la musculaire muqueuse (M). C. Villosité d'intestin grêle. Elle représente un des sites effecteurs essentiels du système immunitaire muqueux. On distingue bien l'épithélium de recouvrement (E) constitué par les cellules hautes ayant une fonction d'absorption et les cellules caliciformes dont la production de mucus (M) est abondante, de la lamina propria (LP) contenant de nombreuses cellules immunitaires. Des lymphocytes intraépithéliaux (LIE) sont visibles entre les cellules épithéliales. La bordure en brosse (BB) des cellules épithéliales fait face à la lumière de l'intestin (L).
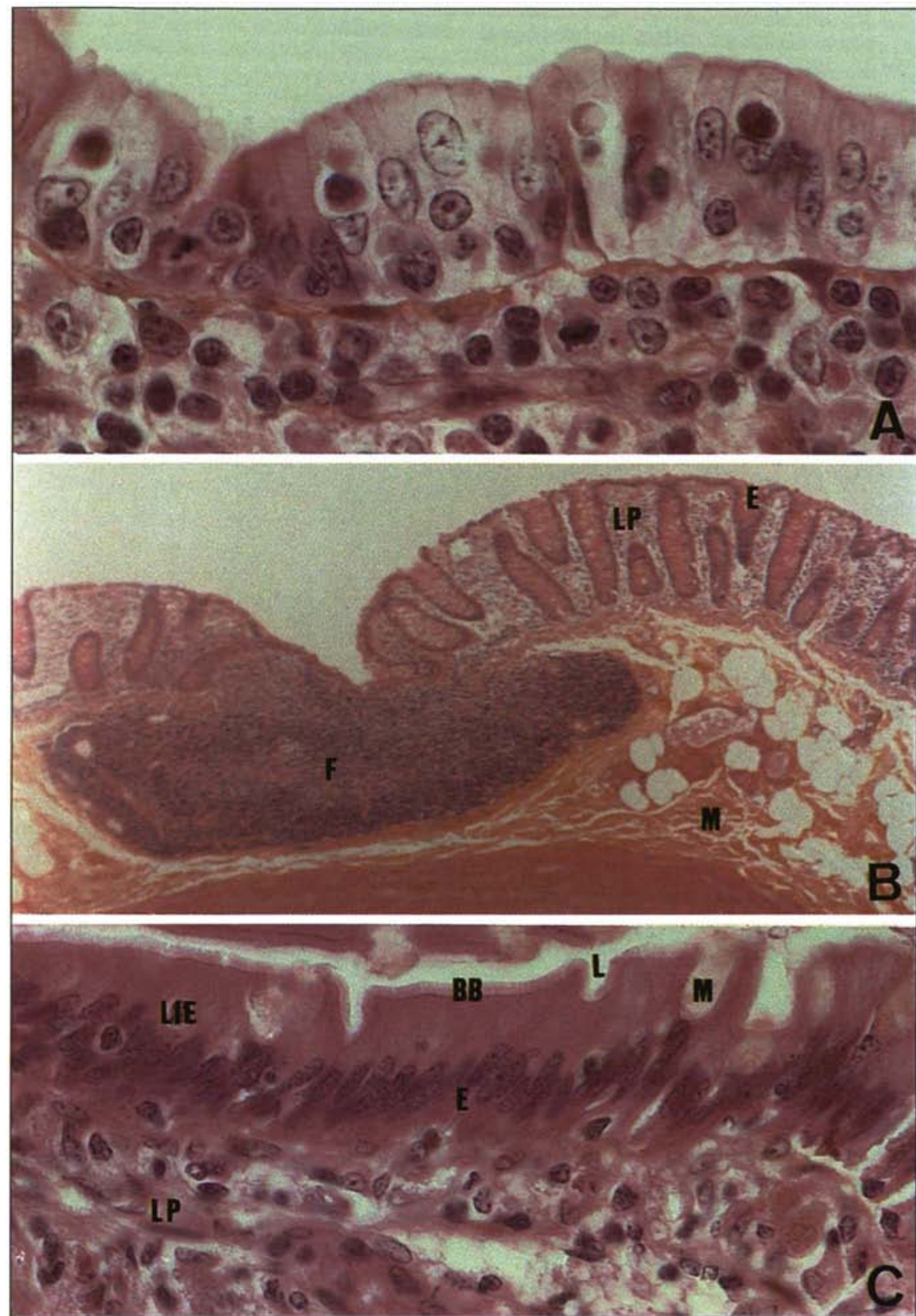

Ces cellules forment une poche intraépithéliale, qui contient des lymphocytes et un petit nombre de macrophages. Les lymphocytes B de la poche intraépithéliale des cellules $M$ sont des cellules immatures (vraisemblablement naïves), exprimant l'IgD en surface, mais pas le marqueur CD45RO. En revanche, les lymphocytes $\mathrm{T}$ de la poche sont mûrs, exprimant tous (:D45RO. ('e sont presque uniquement des lymphocytes $\mathrm{CD}^{+}$ [10]. Les macrophages, enfin, peu- vent mourir rapidement par apoptose lorsqu'ils sont infectés par une bactérie [7], permettant ainsi la libération de cytokines et le déclenchement de la réaction inflammatoire. Des cellules $\mathrm{M}$ peuvent être trouvées dans n'importe quelle portion de l'épithélium, mais elles sont en général concentrées dans des zones spécialisées des épithéliums qui surplombent un follicule lymphoïde de structure très similaire à celle d'un centre germinatif ganglionnaire. On en retrouve dans toutes les voies d'accès à l'organisme (voies respiratoires, voies digestives, urètre pénien, etc.). Dans l'intestin grêle, les follicules sont regroupés, en nombre plus ou moins grand selon l'espèce, dans des plaques de Peyer réparties stratégiquement pour surveiller un bol alimentaire homogénéisé par les mouvements péristaltiques de l'estomac et de l'intestin. Dans le côlon et le rectum, les follicules lymphoïdes sont isolés, ce qui pourrait permettre une 


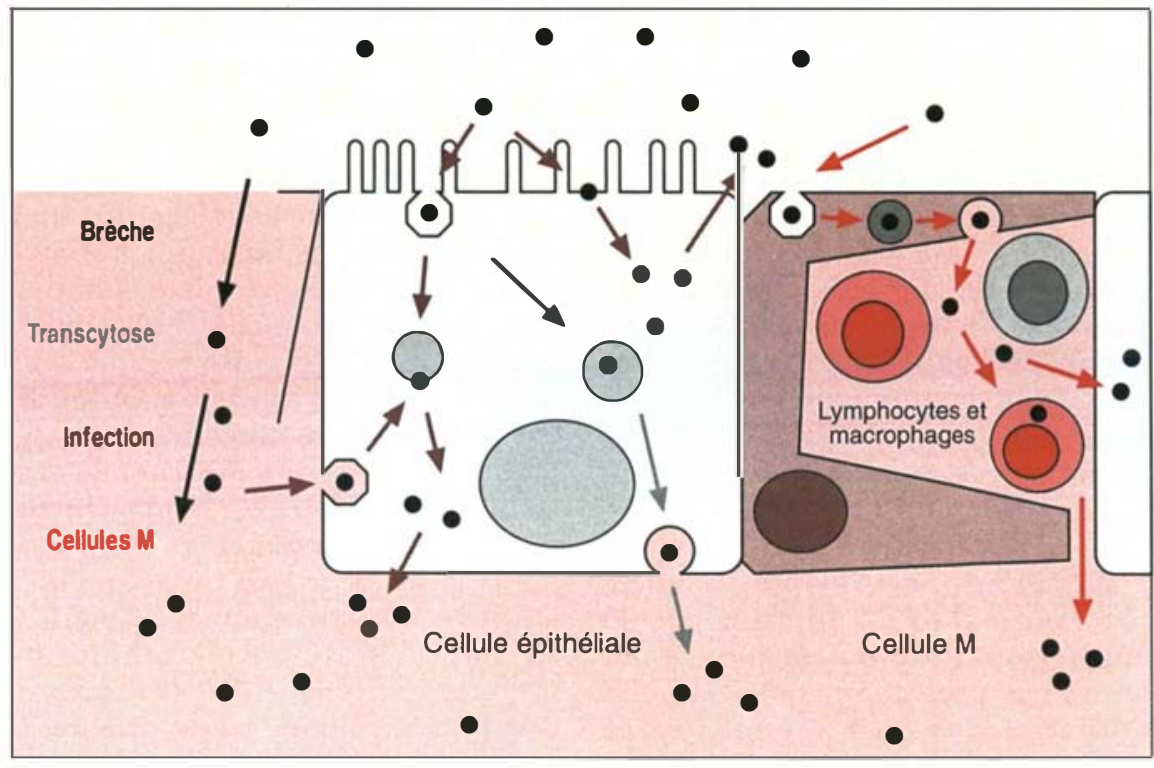

Figure 3. Stratégies de traversée de l'épithélium par un pathogène. Ces stratégies sont générales et modulées en fonction du pathogène. Les virus, par exemple, devront libérer leur acide nucléique dans le cytoplasme pour infecter les cellules, alors que certains parasites se multiplieront à l'intérieur des endosomes ou des lysosomes. La brèche dans l'épithélium (flèches noires) donne au pathogène directement accès, soit à la face basolatérale des cellules épithéliales, ce qui lui permet de les infecter (virus de la stomatite vésiculaire), soit à sa cellule cible (virus de la rage). Une variation de ce thème est le passage de l'agent pathogène entre les cellules épithéliales au cours de la diapédèse de neutrophiles, par exemple. L'infection des cellules épithéliales (flèches bistres) par le domaine apical (paramyxovirus) est un moyen sûr d'infecter l'organisme. Une variante de ce thème est la libération d'une toxine qui interagit avec le domaine apical des cellules épithéliales (toxine cholérique, entérotoxine $d^{\prime} \mathrm{E}$. coli). La transcytose (flèches grises) par les cellules épithéliales non $M$ n'a pas encore été démontrée pour un pathogène. Le transport par les cellules $M$ (flèches rouges) a été démontré pour des virus (MMTV, murine mammary tumor virus), réovirus), pour des bactéries (Shigella, Salmonella), et pour des parasites (Toxoplasma). Le pathogène peut alors, soit infecter les cellules lymphoïdes de la poche épithéliale, soit infecter les cellules épithéliales adjacentes par leur face basolatérale. Les cellules lymphoides infectées peuvent, soit se mobiliser et disséminer ainsi le pathogène dans l'organisme, soit relarguer des cytokines proinflammatoires qui attirent des leucocytes dans la lumière intestinale et permettre ainsi le passage du pathogène entre les cellules épithéliales.

surveillance plus fine de ces sites. Le maintien de ces follicules requiert une activation antigénique permanente par l'exposition aux microorganismes commensaux. Ils sont donc atrophiés chez les animaux axéniques*. L’épithélium associé à ces follicules contient très peu de cellules à mucus et le glycocalyx des cellules épithéliales autres que les cel-

\footnotetext{
*Qui n hébergent aucune esperce microbienne.
} $\mathrm{m} / \mathrm{s} n^{\circ} 5$, wol. 11 , mai 95 poursuivent leur maturation dans les ganglions drainant la muqueuse (ganglions mésentériques dans le cas de l'intestin). Les lymphocytes $T$, quant à eux, se rendent dans le dôme, situé entre le follicule lymphoïde et son épithélium associé, puis dans le ganglion drainant où ils deviennent des lymphoblastes $T$. Les lymphoblastes $T$ et $B$ passent ensuite dans la circulation générale par l'intermédiaire du canal thoracique (figure 1). Ils repeuplent les muqueuses grâce à leur récepteur de domiciliation, l'intégrine $\alpha_{4} \beta_{7}\left(m / s n^{\circ} 10\right.$, vol. 9, p. 1153). L'adressine muqueuse, MadCAM-1, de la superfamille des immunoglobulines et ligand de $\alpha_{4} \beta_{7}$, est présente sur les veinules postcapillaires de l'intestin, mais également au niveau des plaques de Peyer et des ganglions mésentériques.

Le repeuplement des muqueuses se fait d'une manière beaucoup plus ciblée sur le site d'induction que les données initiales ne le laissaient soupçonner [7]. Une immunisation orale che\% les primates donne, par exemple, une réponse essentiellement dans le duodénum et le côlon ascendant, et une immunisation rectale donne une réponse essentiellement rectale [7]. Les réponses induites au niveau des voies respiratoires supérieures restent localisées dans les voies respiratoires. Les réponses induites au niveau de l'intestin grêle peuvent, elles, se redistribuer plus largement (en particulier vers les voies respiratoires). Il y a là une certaine logique, puisque beaucoup d'antigènes respirés sont également avalés. Il pourrait donc y avoir des récepteurs de domiciliation supplémentaires ou une modulation de la rétention locale des lymphocytes. Il est intéressant de penser que cette modulation du repeuplement pourrait provenir d'un effet direct des cellules $\mathrm{M}$ à l'origine de la réponse dans chaque site.

lules $\mathbf{M}$ est réduit. L'ensemble de ces modifications structurales et fonctionnelles facilite l'accès à cet épithélium particulier d'antigènes, de virus ou de bactéries.

La transcytose rapide des antigènes par les cellules $M$ les amène au contact des lymphocytes de la poche intraépithéliale. Les lymphocytes B stimulés se rendent dans le follicule lymphoïde sous-jacent où ils deviennent des lymphoblastes B, puis ils

\section{Effecteurs muqueux}

- Réponse humorale. I.es lymphoblastes B vont pénétrer dans les sites effecteurs comme la lamina propria (ou chorion), mais ils peuvent aussi atteindre des tissus muqueux distants de la voie d'entrée du pathogène grâce à la redistribution par la circulation systémique. Dans ces sites effec- 


\section{RÉFÉRENCES}

11. Cepek KL, Shaw SK, Parker CM, Russell GJ, Morrow JS, Rimm DL, Brenner MB. Adhesion between epithelial cells and $T$ lymphocytes mediated by E-cadherin and the $\alpha_{\mathrm{E}} \beta_{\text {; }}$ integrin. Nature $1994 ; 372: 190-3$.

12. Witkin SS. Immunology of the vagina. Clin Obstet Gynaecol 1993; 36: 122-8.

13. Rocha B, Vassalli P, Guy-Grand D. Thy mic and extrathymic origins of gut in traepithelial lymphocyte populations in mice. Exp Med 1994; 180: 681-6.

14. Panja A, Blumberg RS, Balk SP. Maye L. CDld is involved in $\mathrm{T}$ cell-epithelial cel interactions. $\int$ Exp Med 1993; 178: 1115-9.

15. Karapetian O, Shakhov AN, Kraehenbühl JP, Acha-Orbea H. Retroviral infec tion of neonatal Peyer's patch lymphocytes the mouse mammary tumour virus model. $j$ Exp Med 180: 1511-6.

16. Lafon M. Superantigènes viraux. médecine/sciences $1994 ; 10: 78-81$.

17. Perdomo JJ, Gounon P, Sansonetti PJ Polymorphonuclear leukocyte transmigration promotes invasion of colonic epithelial monolayer by Shigella flexneri. I Clin Invest $1994 ; 93: 633-43$

18. Sansonetti PJ, Mounier J, Prévost MC, Mège RM. Cadherin expression is required for the spread of Shigella flexneri between epithelial cells. Cell 1994; 76: 829-39.

19. Lehner T, Bergmeier LA, Tao L, Panagiotidi C, Klavinskis LS, Hussain L, Ward G Meyers N, Adams SE, Gearing AJH, Brookes $R$. Targeted lymph node immunization with simian immunodeficiency virus p 27 antigen to elicit genital, rectal and urinary immune responses in nonhuman primates. I Immunol $1994 ; 1858-68$.

20. Mazanec MB, Kaetzel CS, Lamm ME, Fletcher D, Nedrud JG. Intracellular neutralization of virus by immunoglobulin A antibodies. Proc Natl Acad Sci USA 1992; 89 : 6901-5.

21. Brousse $\mathrm{N}$. Le système immunitaire de la muqueuse digestive. Barrière digestive et im- teurs muqueux, les cellules B vont se différencier en plasmocytes, en particulier à $\operatorname{IgA}$. Ce circuit de distribution des IgA à partir des tissus inducteurs comme le GALT (gut associated lymphoid tissue) ou le BALT (bronchus associated lymphoid tissue) vers les sites effecteurs des Ig A comme la lamina propria de l'intestin, des bronches ou du tractus génital a donc été appelé le système immunitaire muqueux à médiation humorale [9].

Les plasmocytes à $\operatorname{IgG}$ et à $\operatorname{IgM}$ sont moins abondants dans le chorion que les plasmocytes à $\operatorname{IgA}$. Ces immunoglobulines ne sont pas, ou peu, sécrétées dans la lumière de la muqueuse, et pourraient donc jouer un rôle de protection contre la dissémination des pathogènes, après qu'ils aient franchi l'épithélium. Les IgA sécrétoires (S-IgA) produites localement sont parmi les éléments les plus importants de la réponse humorale protectrice au niveau des muqueuses [1-3]. Cet isotype constitue, en effet, plus de $80 \%$ de tous les anticorps produits au niveau des muqueuses, mais le plus important est certainement que ces S-IgA sont induites, transportées et réglées par des mécanismes complètement différents de ceux qui interviennent dans les réponses immunitaires humorales systémiques. Une fois transportées à la surface de la muqueuse, les S-IgA peuvent lier les antigènes, former des complexes immuns et bloquer l'interaction de ces antigènes avec la surface muqueuse, exerçant ainsi un mécanisme d'immunité protectrice que l'on appelle l'exclusion immune. Les IgA présentes dans les sécrétions externes sont essentiellement dimériques ou tétramériques. Cette multivalence les rend capables de mieux agglutiner les bactéries que des Ig monomériques. Outre les virus, les IgA sécrétoires sont capables de neutraliser d'autres antigènes biologiquement actifs, comme les toxines bactériennes et les enzymes. Ces IgA fonctionnent dans un environnement très riche en enzymes protéolytiques et l'une de leurs caractéristiques principales est la résistance à la protéolyse. Cette dernière est renforcée par l'association à la pièce sécrétoire et permet de conserver un grand nombre des fonctions biologiques liées aux molécules Ig intactes.
- Réponse cellulaire. Il faut noter, toutefois, que ces tissus sont aussi capables de développer une réponse immunitaire à médiation cellulaire. Certains lymphoblastes $\mathrm{T}$ se rendent dans l'épithélium lui-même. Ce sont les lymphocytes intraépithéliaux (LIE). Ils sont ancrés dans l'épithélium grâce à l'interaction entre l'intégrine $\alpha_{1} \beta_{i}(C D 103$, aussi connue sous le nom d'HML-1) et la cadhérine $E$. Cette molécule d'adhérence homotypique de la membrane latérale des cellules épithéliales est fondamentale pour la morphogenèse des épithéliums et la formation de la jonction étroite (ou barre terminale) qui assure l'étanchéité des épithéliums simples. La découverte d'une interaction hétérotypique de la cadhérine $\mathrm{E}$ ayant un rôle dans l'adhérence des LIE est donc remarquable [11].

Les LIE sont essentiellement $\mathrm{CD}^{+}$ [1] dans l'intestin et dans le vagin [12]. En revanche, on trouve dans les voies respiratoires une proportion de lymphocytes $\mathrm{CD}^{+}$plus proche de celle du sang. Parmi les LIE intestinaux, il existe une population indépendante du répertoire thymique [13], qui peut exprimer un récepteur T de l'antigène $\gamma \delta\left(\mathrm{m} / \mathrm{s} n^{\circ} 4\right.$, vol. 610 , p. 483), et/ou une molécule CD8 $\alpha \alpha$. Les LIE expriment fréquemment des marqueurs d'activation. Ils ne semblent pas subir de délétion clonale pour les autoantigènes. Des fonctions trophiques, suppressives (surtout pour les lymphocytes $\gamma \delta$ ) et cytotoxiques ont toutes été évoquées pour les LIE $\left(\mathrm{m} / \mathrm{s} n^{\circ} 5\right.$, vol. $\left.10, p .603\right)$, mais c'est une activité cytotoxique que requiert la défense de l'organisme. Une telle activité dirigée contre le réovirus a été mise en évidence dans l'intestin chez la souris. De même, la guérison d'une infection par le virus influenza des voies respiratoires inférieures passe par des lymphocytes $\mathrm{T}$ cytotoxiques. Les LIE peuvent reconnaître des molécules d'histocompatibilité non classiques exprimées sur la face basolatérale des cellules épithéliales, ayant des similitudes avec les molécules du CMH I [14].

Les autres lymphoblastes se rendent dans le chorion. Les lymphoblastes $\mathrm{T}$ se différencient en lymphocytes du chorion (LLP), avec un rapport CD4/CD8 proche de celui du sang. Leur spécificité muqueuse est appa- 


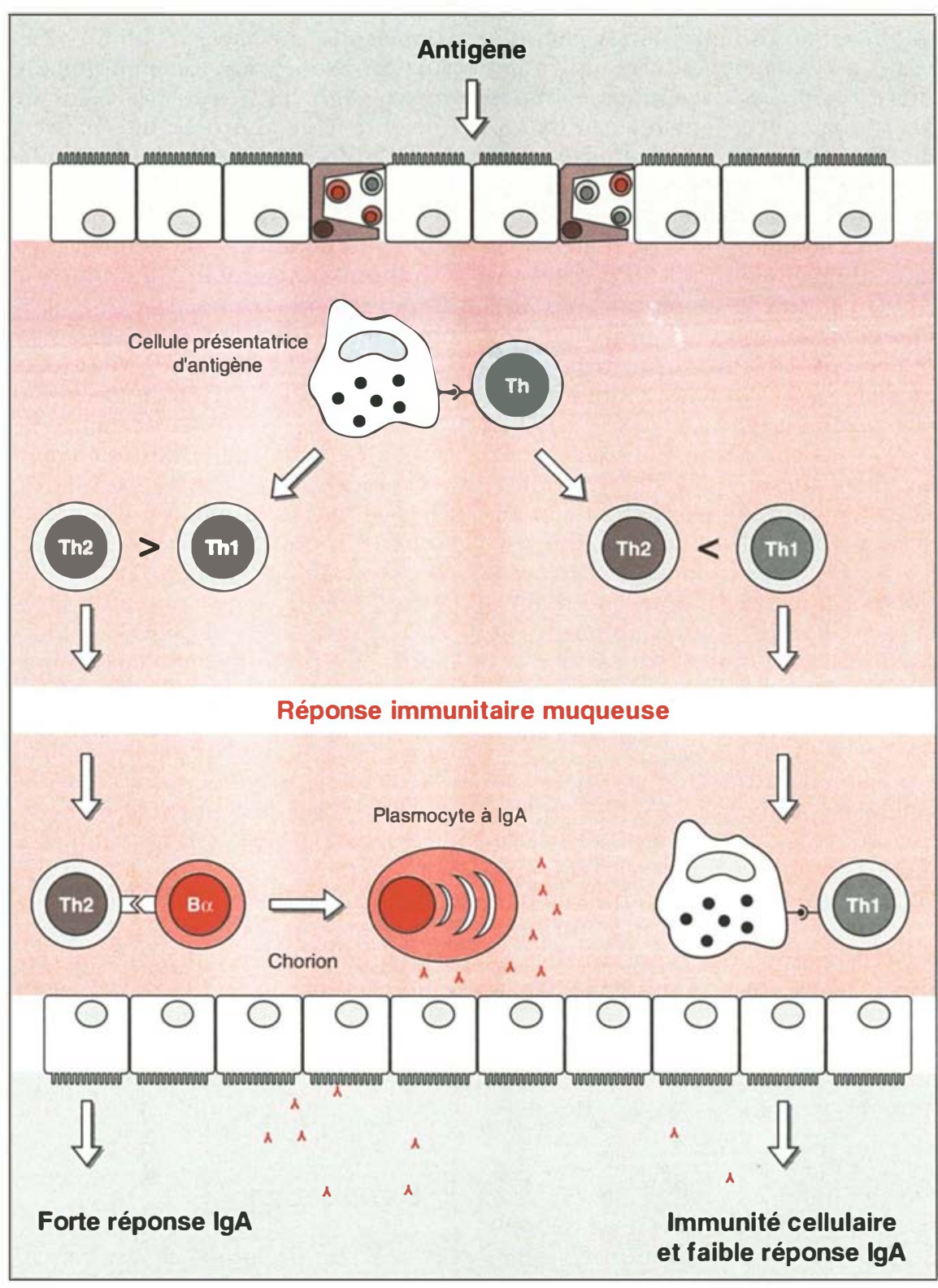

Figure 4. Polarisation de la réponse immunitaire muqueuse. Selon la présentation de l'antigène mis en présence des cellules $M$, les lymphocytes $T$ auxiliaires s'orienteront vers un phénotype Th1, ou vers un phénotype Th2. Le prototype de l'antigène stimulant une réponse de type Th1, c'est-à-dire essentiellement l'immunité cellulaire, est le parasite Toxoplasma gondii. $C^{\prime}$ est pourquoi un certain nombre de protocoles vaccinaux expérimentaux utilisent des vecteurs vivants recombinants pour essayer de développer l'immunité cellulaire antivirale, par exemple. Le prototype de l'antigène stimulant une réponse de type Th2 (c'est-à-dire une forte sécrétion d'lgA dans la lumière intestinale) est la toxine cholérique soluble. La régulation fine de ces réponses est encore moins bien comprise au niveau de l'immunité locale qu'au niveau de l'immunité systémique. (Modifiée d'après [3].) rente dans leur expression de l'intégrïne $\alpha_{E} \beta_{7}$ à faible niveau ou de l'intégrine $\alpha_{4} \beta_{7}$. Il est vraisemblable qu'ils expriment des activités fonctionnelles voisines de celles des lymphocytes périphériques.

\section{Relations pathogène- système immunitaire muqueux}

Les pathogènes se sont adaptés au système immunitaire muqueux, et le détournent pour pouvoir envahir leur hôte. Deux exemples sont particulièrement représentatifs. Le MMTV (murine mammary tumor virus) pénètre dans l'ensemble de la muqueuse intestinale chez le souriceau nouveau-né, mais c'est seulement au niveau des plaques de Peyer qu'il peut infecter les lymphocytes $B$ et $\mathrm{T}$ de la poche intraépithéliale des cellules M [15]. Ces lymphocytes sont à l'origine de la dissémination du virus à l'ensemble de l'organisme et, en particulier, au tissu cible, la glande mammaire. Le superantigène du MMTV facilite sa dissémination en lui permettant d'échapper à la réponse immunitaire et de persister dans son hôte [16]. La shigelle a une stratégie invasive plus subtile: transporté par les cellules $\mathbf{M}$, ce microorganisme infecte les macrophages de la poche intraépithéliale. L'ILl relarguée lors de l'apoptose de ces macrophages attire des neutrophiles dans la muqueuse intestinale [7]. En traversant la muqueuse, ces neutrophiles perturbent l'étanchéité des jonctions étroites, permettant ainsi aux shigelles de se retrouver sur la face latérale des cellules épithéliales [17]. C'est là qu'elles rencontrent leur récepteur, qu'elles infectent les cellules épithéliales, puis se propagent de cellule en cellule grâce aux cadhérines [18]. Ce mécanisme est très rapide, et conduit en quelques heures à la destruction de l'épithélium intestinal $\left(\mathrm{m} / \mathrm{s} n^{\circ} 1\right.$, vol. 11 , p. 128).

\section{Applications vaccinales}

Il est tout à fait étonnant qu'en dépit de notre connaissance du système immunitaire local, presque tous les vaccins actuellement utilisés chez l'homme soient donnés par voie parentérale. L'immunisation systé- 
mique est particulièrement inefficace pour l'induction d'une réponse immune locale. Dans la mesure où la majorité des micro-organismes infectieux sont rencontrés au niveau de la surface muqueuse, il est important de considérer que l'induction d'une réponse protectrice doit se produire au niveau des tissus muqueux. La compartimentalisation des réponses implique que chaque muqueuse cible doive être protégée par une immunisation spécifique, notamment en ce qui concerne l'immunité à médiation cellulaire, et qu'il ne peut exister de "vaccin oral" protégeant l'ensemble des muqueuses accessibles à un pathogène donné $(\mathrm{m} / \mathrm{s}$ $n^{\circ} 8$, vol. 7,1 . 874). La nature de la réponse locale pourrait aussi se traduire par une spécificité antigénique dépendant d'un site inducteur précis. Par exemple, une injection à proximité des ganglions iliaques semble induire une bonne réponse immunitaire à la fois dans le rectum et dans les voies génitales [19], et cependant, la nature des épitopes reconnus par les lymphocytes $\mathrm{T}$ auxiliaires est différente dans chacun de ces sites. Par ailleurs, une seule immunisation systémique suffit à inhiber partiellement la réponse immunitaire locale, probablement par le passage d'anticorps sériques à faible dose dans les lumières par transsudation. Les protocoles vaccinaux devront donc être modulés en conséquence. De nombreuses stratégies vaccinales essayent de stimuler la réponse Ig A (figure 4). Celle-ci est primordiale dans la protection de l'intestin et des voies respiratoires. Ces IgA sécrétées ont un rôle essentiel dans la protection de l'organisme contre l'invasion par les commensaux de l'intestin, et contre les infections virales et bactériennes. La prévention de l'infection des voies respiratoires supérieures par le virus influenza se fait par les Ig A sécrétoires. Et il a été montré in vitro que la protection des cellules épithéliales contre la lyse par le virus influenza peut se faire par les IgA intracellulaires au cours de leur transcytose [20].

D'une façon générale, il est très difficile d'induire une réponse immunitaire à médiation humorale impliquant des IgA sécrétoires lorsqu'on utilise une immunisation orale par

sation de doses importantes par voie orale peut même induire une tolérance orale. Les exceptions connues à ce jour concernent les exotoxines de $V$. cholerap et une entérotoxine d'L. coli. Les caractéristiques de ces protéines sont de pouvoir interagir avec les gangliosides, et d'élever le taux intracellulaire d'AMPc. Elles induisent toutes les deux une réponse muqueuse lorsqu'elles sont données oralement, ce qui en fait de remarquables adjuvants et immunogènes au niveau local $\left(\mathrm{m} / \mathrm{s} n^{\circ} 8\right.$, vol. 7 , p. 874). Cependant l'utilisation de bactéries atténuées ou de vecteurs viraux semble plus prometteuse pour la mise au point de vaccins ciblés au niveau des sites inducteurs de la réponse muqueuse. L'efficacité de cette approche, démontrée initialement par les vaccins oraux de la poliomyélite, devra être confirmée dans le cas d'autres pathogènes ayant un tropisme pour les muqueuses.

Les essais de protection contre la shigelle sont mitigés [7] : il y a de très nombreux sérotypes et les souches atténuées induisent peu de réponse alors que les souches induisant une réponse protectrice sont à l'origine d'effets secondaires importants (diarrhée). Le vaccin oral contre le choléra est en meilleure voie [7]. Une souche atténuée, dont la toxine A est délétée et la toxine $B$ intacte, est très immunogène dans les pays développés, beaucoup moins dans les pays sous-développés. La protection est seulement de $75 \%$, mais elle est totale contre les formes sévères du choléra. La nouvelle formulation comporte les trois souches rencontrées au niveau mondial.

L'utilisation des bactéries recombinantes atténuées a été développée intensément ces dernières années. De nombreuses études se sont focalisées sur l'utilisation de ces systèmes bactériens pour exprimer des épitopes, cibles de la réponse immunitaire contre des protéines hétérologues provenant de pathogènes. L'intérêt de ces bactéries est de permettre une vaccination par une seule immunisation. L'une des approches a consisté à intégrer dans le chromosome de Salmonella typhimirium le gène codant pour la protéine vaccinale considérée sous la forme d'une protéine de fusion. La production continuelle pendant plusieurs jours de la protéine chimérique permet d'obtenir l'induction d'une réponse immunitaire de type $\operatorname{Ig} \mathrm{A}$ au niveau des tissus inducteurs. Une approche alternative a été d'utiliser une souche recombinante de Salmonella exprimant un antigène sous le contrôle d'un promoteur inductible uniquement en condition anaérobie. L'induction d'une réponse protectrice est obtenue par une simple dose.

A côté des systèmes bactériens, des systèmes viraux ont été utilisés. Par exemple, les vecteurs adénovirus recombinants pouvant exprimer, soit l'enveloppe, soit la protéine Gag du VIH, ont été récemment utilisés pour tenter d'induire une réponse anticorps anti-VIH par voie orale. Les résultats, bien que préliminaires, laissent envisager que ces vecteurs pourraient prendre une place importante dans les protocoles d'immunisation muqueuse. Des recombinants influenza semblent être également des vecteurs prometteurs: ils permettent d'obtenir une protection contre Plasmodium assurée par les cellules T cytotoxiques et un titre élevé d'IgA contre la gpl60 de VIH [7].

Parallèlement aux organismes vivants atténués, l'utilisation de microsphères plus ou moins rapidement biodégradables permettrait d'obtenir à la fois une immunisation de type primaire et une immunisation de type de rappel en utilisant une seule dose de vaccination.

\section{Conclusion}

Le système immunitaire est remarquablement modulé par la relation hôte-pathogène: l'organisme élabore des stratégies de défense locale de plus en plus complexes au fur et à mesure que les pathogènes développent des stratégies de plus en plus subtiles pour contourner ces mécanismes de défense en les utilisant dans leurs stratégies invasives. Un équilibre s'établit entre le pathogène et l'hôte, qui doit être pris en compte dans toute stratégie de protection

\section{TIRÉS À PART}

C. Butor 


\section{Summary}

Local immunity and vaccination

The organism is protected against the entry of microorganisms by a barrier constituted by a series of mechanisms. Passive mechanisms include tightness of the epithelia lining the body and carbohydrates (mucus, surface glycoconjugates). Defensins and the inflammatory response represent active, but not specific, protection. The specific mucosal immune system comes into play when these non-specific mechanisms have failed. The mucosal immune response is initiated in lymphoid follicles and their associated epithelium. Rapid transcytosis of antigens is the task of specialized cells in the follicle associated epithelium: the $\mathbf{M}$ cells. $\mathbf{M}$ cells differ from site to site and from species to species, and could differenciate locally from other epithelial cells.
Antigen is brought in contact with the $\mathrm{T}$ and $\mathrm{B}$ cells present in a pocket formed by the $M$ cells. The stimulated blasts migrate to the underlying follicle and the draining lymph nodes, where they mature, then move on to the bloodstream. The integrin $\alpha_{4} \beta_{7}$ allows them to home to the mucosa. Other receptors could be involved in fine tuning the homing site, which is more centered on the induction site than previously thought. B cells differenciate into plasma cells (mainly $\operatorname{Ig} \mathrm{A}$ ) in the lamina propria. The IgA transcytosed by the epithelium serve in immune exclusion, intracellular viral neutralisation and export of antigen back to the lumen. The IgA response is the major protective response in the mucosal immune system, and is the one that mucosal vaccines attempt to stimulate. T cells home either to the lamina propria (lamina propria lymphocytes, LPL) or to the epithelium (intraepithelial lymphocytes, IEL). Other IEL come directly from the bone marrow. Gut IEL are mostly CD8 ${ }^{+}$and $\alpha_{\mathrm{F}} \beta_{7}{ }^{+}$. Their functional activities include cytoxicity. The mouse-mammary tumor-virus MMTV (which disseminates via the entero-enteric cycle), and Shigella (which takes advantage of the inflammatory response to weaken the epithelial barrier), are examples of pathogens "highjacking " the mucosal immune response in their invasive strategies. This interplay of host and pathogen, as well as the compartmentalization of the mucosal immune system, will have to be addressed by vaccinal strategies. 